\title{
Amplitude Control of Phase Modulation for Dithered Closed-loop Fiber Optic Gyroscope
}

\author{
Kyoung-Ho Chong ${ }^{1 *}$, Kil-To Chong ${ }^{2}$, and Young-Chul Kim $^{3}$ \\ ${ }^{1}$ Agency for Defense Development, Taean 357-900, Korea \\ ${ }^{2}$ Chonbuk National University, Jeonju 561-756, Korea \\ ${ }^{3}$ Kunsan National University, Kunsan 573-701, Korea
}

(Received July 17, 2012 : revised October 12, 2012 : accepted October 15, 2012)

\begin{abstract}
The amplitude error of phase modulator used in closed-loop fiber optic gyroscope has occurred by the temperature dependency of the electro-optic coefficient, and also can be due to the square-wave dither signal which is generally applied for eliminating the deadzone. This error can cause bias drift and scale factor error. This paper analyzes the temperature dependency of the modulation amplitude and the relationship with the scale factor of the gyroscope, and deals with an amplitude control method. The error calculation logic considering the dither signal is implemented on the signal processing module. The result of experiments from a prototype gyroscope shows the effect of the modulation amplitude control and a considerable improvement on performances.
\end{abstract}

Keywords: Phase modulation, Integrated optic circuits, Fiber optic gyroscope

OCIS codes : (130.0130) Integrated optics; (060.0060) Fiber optics and optical communications; (280.0280) Remote sensing and sensors

\section{INTRODUCTION}

The introduction of the Fiber Optic Gyroscope(FOG) occurred in the late 1970s after the invention of optical fibers. With help received from the researchers in the field of interferometry and telecommunications, The FOG has proven to be a leading technology in various applications for imaging systems, antennas, precise aiming of telescopes, and navigation systems [1-5].

The FOG is a mature technology and was originally designed as a low-cost alternative to the Ring Laser Gyroscope [2-3]. Due to the solid-state optical configuration and fiber technology, the FOG's performance could lead to $0.001 \mathrm{deg} / \mathrm{hr}$ stability in miniature design [4-5]. The main optical components of FOG are composed of fiber, integrated optic chip(IOC), coupler, light source, and detector.

One of the key elements in determining the bias and scale factor of FOG is the modulator's characteristics in the IOC $[6,11-12]$. The main function of the IOC is to split the light and to modulate the phases which are combined in a $\pi / 2$-biased phase for maximum sensitivity, Sagnac phase shift by input rate, and feedback phase for compensating the rate. The important parameters of the
IOC for improving the performance of gyroscope are polarization crosstalk, polarization extinction ratio, and modulation amplitude efficiency. Among them, the required level of polarization crosstalk and extinction ratio can be achieved by the manufacturing process.

However, as the modulation amplitude efficiency is intrinsically induced by temperature variation, controlling of the modulation amplitude should be needed because the bias and scale factor are strongly dependent on the feedback element which is directly proportional to the modulation amplitude coefficient of the IOC [7-13]. In addition, the modulation amplitude error can also occur due to the square-wave dither signal which is composed of plus and minus virtual rate. This error can cause bias drift and scale factor error in a closed-loop FOG.

This paper analyzes the temperature dependency of phase modulation amplitude from the effective electro-optic coefficient. In order to investigate how the modulation amplitude error affects the gyroscope, the relationship between temperature dependency of the phase modulation amplitude and the scale factor of the gyroscope is investigated. The error calculation method is improved for the gyroscope with the square-wave dither signal, and implemented on the signal

\footnotetext{
*Corresponding author: khchong@add.re.kr

Color versions of one or more of the figures in this paper are available online.
} 
processing module. Some of experimental results with and without the amplitude control loop are also discussed.

\section{A BASIC OPERATION OF FIBER OPTIC GYROSCOPES AND PHASE MODULATION AMPLITUDE ERROR}

The FOG is based on the Sagnac effect, which produces a phase difference proportional to the rotation rate [1]. Light from the broadband source is directed through the first coupler and a single-mode filter to a second coupler included on the IOC. The second coupler divides the light into two parts: one that will propagate clockwise through the sensing coil, and the other that will propagate counterclockwise. They have opposite phase shift sensitivities to rotation about the coil axis. The Sagnac phase difference between clockwise and counter-clockwise becomes :

$$
\Delta \phi_{R}=\frac{2 \pi L D}{\lambda c} \cdot \Omega=K_{O S F} \cdot \Omega
$$

where $\lambda$ is the central wavelength of the light source ; $L$ is the length of fiber ; $D$ is the average loop diameter of the fiber; $\Omega$ is the input rate ; $\Delta \phi_{R}$ is the Sagnac phase shift ; $K_{O S F}$ is the optical scale factor of gyroscope.

Figure 1 shows the general configuration of optics and IOC in FOG.

The phase difference induced by input rate is expressed as a cosine relationship for optical power. Once the broadband source generates the power, the photodector output through Sagnac loop in fiber spool $P_{d}$ is expressed as :

$$
P_{d}=\frac{P_{0}}{2}\left(1+\cos \Delta \phi_{R}\right)
$$

where $P_{0}$ is the peak power of the photodetector output.

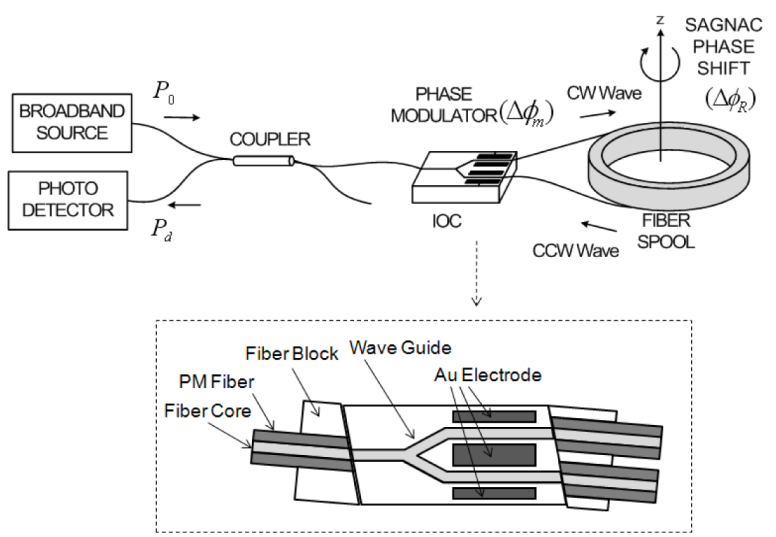

FIG. 1. Configuration of FOG optics and the structure of the IOC.
To maximize the sensitivity of the gyroscope, the square-wave modulation-demodulation method is commonly used [12-14]. When the square-wave signal with $\Delta \phi_{m}$ amplitude is generated, the photodetector output during a period is expressed as :

$$
\begin{aligned}
& P_{1}=\frac{P_{0}}{2}\left[1+\cos \left(\Delta \phi_{R}+\Delta \phi_{m}\right)\right] \\
& P_{2}=\frac{P_{0}}{2}\left[1+\cos \left(\Delta \phi_{R}-\Delta \phi_{m}\right)\right] \\
& P_{\text {rate }}=P_{1}-P_{2}=P_{0} \sin \Delta \phi_{m} \sin \Delta \phi_{R} \approx P_{0} \Delta \phi_{R}
\end{aligned}
$$

where $\Delta \phi_{R}$ is small ; $\Delta \phi_{m}$ is $\pi / 2$.

When the square-wave modulation is applied and the input rate occurs, the photodetector output signal is shown in Fig. 2.

If $P_{0}$ is $1, P_{\text {rate }}$ is simply equal to $\Delta \phi_{R}$. The equation (5) is only available if both $P_{0}$ and $\Delta \phi_{m}$ are stable. When the phase modulator has a small amplitude error, the equation (5) can be replaced $\Delta \phi_{m}$ with $\Delta \phi_{m}+\delta$ :

$$
P_{\text {rate }}=P_{1}-P_{2}=P_{0} \sin \left(\Delta \phi_{m}+\delta\right) \sin \Delta \phi_{R} \approx P_{0} \cos \delta \Delta \phi_{R}
$$

where $\Delta \phi_{R}$ is small ; $\Delta \phi_{m}$ is $\pi / 2$.

From the equation (6), photodetector output has parasitic error due to a nonlinear term $\cos \delta$, and can fluctuate due to environmental change. Fig. 3 shows the photodetector output when the phase modulation amplitude error occurs.

In order to reduce the error, the temperature characteristics

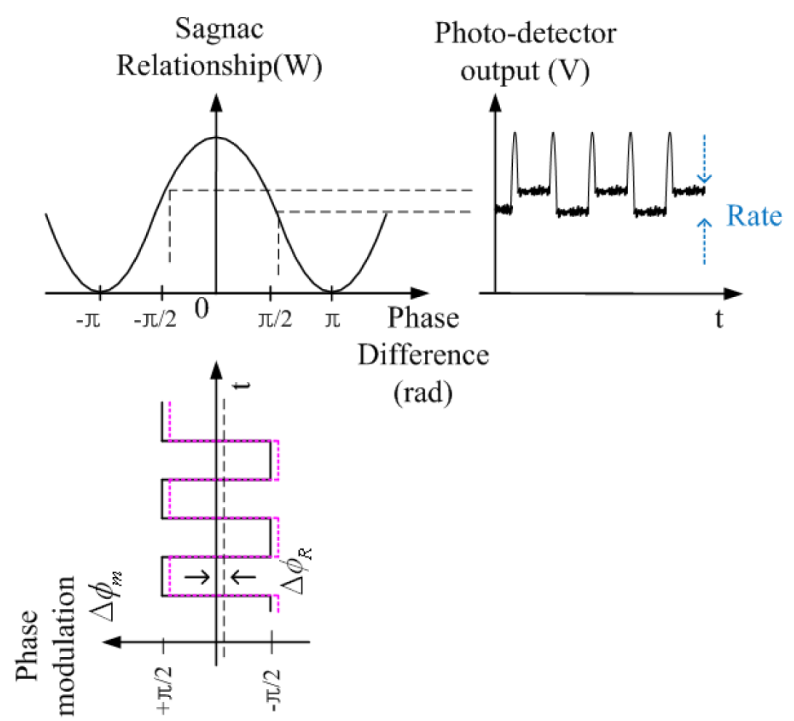

FIG. 2. Phase modulation and photodetector output when input rate occurs. 
of the phase modulator should be analyzed first. The structure of the phase modulator is shown in Fig. 1. The modulator has two electrodes which can double the modulation efficiency. When one electrode of $\mathrm{LiNbO}_{3}$ is modulated, the phase modulation amplitude becomes [7] :

$$
\Delta \phi_{m}=\gamma \Delta n \frac{2 \pi}{\lambda} L_{m}
$$

where $\gamma$ is the optical confinement factor, defined as the portion of optical mode that is confined in the active layer ; $L_{m}$ is the modulation length ; $\Delta n$ is the optical index change in the waveguide active layer.

Assume that the optical wavelength is constantly stabilized by a thermo-electric cooler. The temperature dependency of phase modulation amplitude $d\left(\Delta \phi_{m}\right) / d T$ is proportional to the temperature dependency of the optical index $d(\Delta n) / d T$ because $\gamma$ and $L_{m}$ are relatively small [7-8].

Here, we should look into the optical index change in detail. When the voltage $V$ is applied to the electrodes on the modulator, the optical index change can be expressed as [7]:

$$
\Delta n=\frac{1}{2} n_{e f f}^{3} r_{e f f} \frac{V}{d}
$$

where $n_{\text {eff }}$ is the effective optical index of the active layer ; $r_{\text {eff }}$ is the effective electro-optic coefficient; $V$ is the applied voltage ; $d$ is the spatial gap between the electrodes across which the voltage is applied.

From equation (8), the temperature dependency of optical index is composed of $n_{e f f}$ and $r_{e f f}$. The reports shows that the temperature dependency of $r_{\text {eff }}$ is approximately $4.9 \times 10^{-4}$

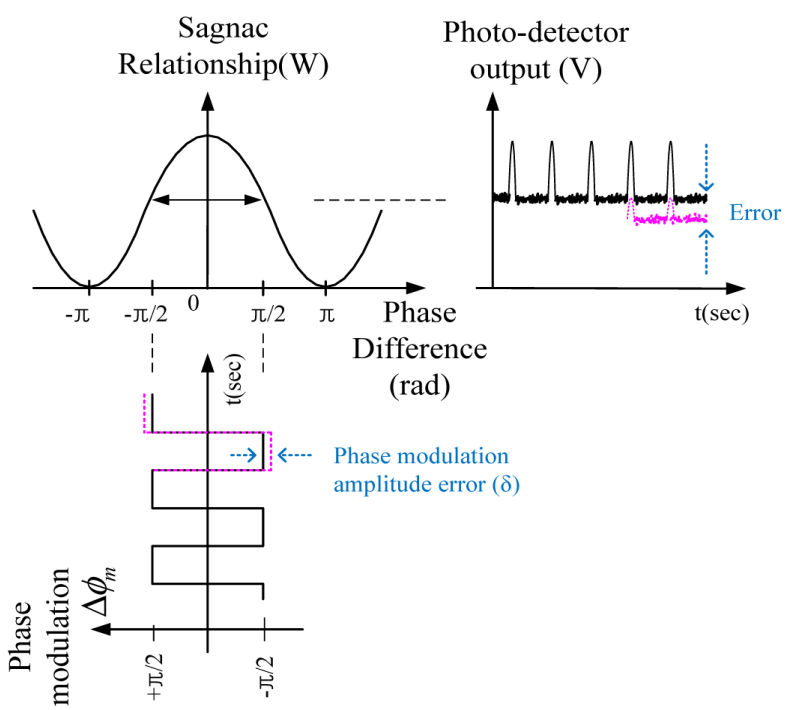

FIG. 3. Photodetector output with $\pm \pi / 2$ modulation when the modulation amplitude error occurs.
$/{ }^{\circ} \mathrm{C}$ and the temperature dependency of $n_{\text {eff }}$ is approximately $4.0 \sim 5.0 \times 10^{-5} /{ }^{\circ} \mathrm{C}[7-8]$.

Because the temperature dependency of $r_{e f f}$ is typically ten times higher than the temperature dependency of $n_{e f f}$, combining equation (7) and (8), the temperature dependency of phase modulation amplitude can be written as :

$$
\frac{d\left(\Delta \phi_{m}\right)}{d T} \approx \frac{d(\Delta n)}{d T} \approx \frac{d r_{e f f}}{d T}, \quad\left(\frac{d r_{e f f}}{d T}>>\frac{d n_{e f f}}{d T}\right)
$$

\section{A MODULATION AMPLITUDE CONTROL METHOD}

In this section, the error calculation method from the photodetector output will be derived and an improved method will be suggested. A control loop modeling and the relationship between temperature dependence of phase modulation amplitude and scale factor of a gyroscope will be discussed next.

It is impossible to find the phase modulation error from the $\pm \pi / 2$ biasing modulation alone because we don't know the reference power level of the photodetector output, as shown in Fig. 3. In order to find the phase modulation error, it is necessary to generate additional modulation, \pm 3 $\pi / 2$. Fig. 4 shows the photodetector output with both $\pm \pi / 2$ and $\pm 3 \pi / 2$ modulation.

The modulation steps(pulses) come from the D/A converter through the digital steps determined by the digital register. The closed loop signal processing uses both $\pm \pi / 2$ biasing modulation and ramp modulation. The ramp modulation integrated by input rate digitally is used as feedback phase, which compensate the Sagnac phase proportional to the input rate. Typically, the maximum total steps, combined bias and ramp modulation, are limited to $2 \pi$ because the maximum output voltage of the $\mathrm{D} / \mathrm{A}$ converter is set to 2

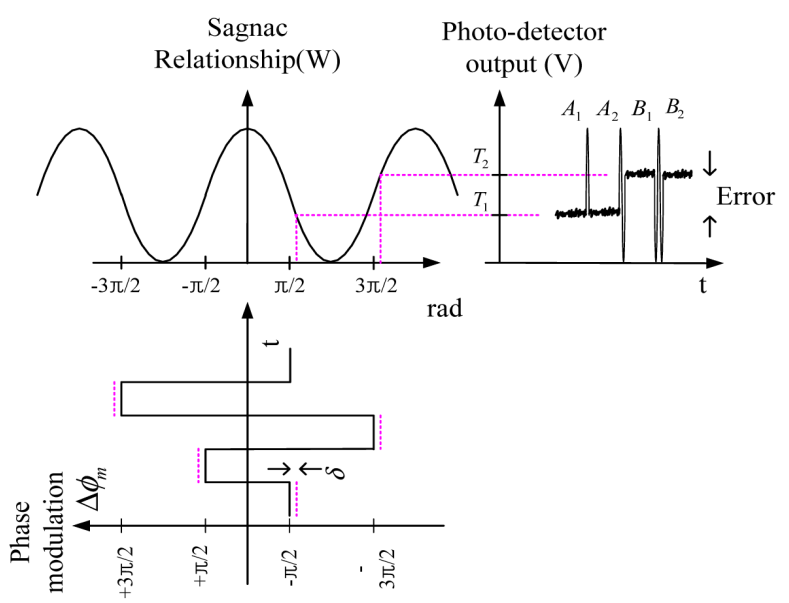

FIG. 4. Photodetector output with both $\pm \pi / 2$ and $\pm 3 \pi / 2$ modulation when the modulation amplitude error occurs. 
$\pi$. When total steps reach the $2 \pi$-limit, the step register is reset, and $\pm \pi / 2$ biasing modulation is changed into $\pm 3 \pi / 2$ automatically. Considering the modulation amplitude error $\delta$, the photodetector output before and after $2 \pi$-reset can be expressed as :

$$
\begin{aligned}
P_{1} & =\frac{P_{0}}{2}\left[1+\cos \left(\Delta \phi_{m}+\delta\right)\right] \\
P_{2} & =\frac{P_{0}}{2}\left[1+\cos \left(2 \pi-\Delta \phi_{m}+\delta\right)\right] \\
& =\frac{P_{0}}{2}\left[1+\cos \left(\Delta \phi_{m}-\delta\right)\right]
\end{aligned}
$$

The modulation amplitude error can be expressed by subtracting $P_{1}$ from $P_{2}$.

$$
P_{\text {error }}=P_{2}-P_{1}=P_{0} \sin \Delta \phi_{m} \sin \delta \approx \delta
$$

In practice, the modulation amplitude error is calculated by the difference between average of $\pm \pi / 2$ steps and average of $\pm 3 \pi / 2$ steps in order to minimize the noise. The steps can increase or decrease with the input rate value. For a low rate, the steps increase due to a long modulation period that takes long for a small ramp bit to accumulate until $2 \pi$. Likewise, the steps decrease in a high rate and vary quickly from $\pm \pi / 2$ steps to $\pm 3 \pi / 2$ steps. Applying averaging method, the modulation amplitude error can be expressed as :

$$
\delta=\frac{1}{m} \sum_{j=1}^{m} B_{j}-\frac{1}{n} \sum_{i=1}^{n} A_{i}
$$

$n:$ number of photodetector outputs for $\pm \pi / 2$ modulation step

$m$ : number of photodetector outputs during $\pm 3 \pi / 2$ step

$A_{i}$ : photodetector output $\left(P_{1 i}\right)$ for $\pm \pi / 2$ modulation step

$B_{j}$ : photodetector $\operatorname{output}\left(P_{2 j}\right)$ for $\pm 3 \pi / 2$ modulation step

This equation is commonly used because it can be simply calculated [13-14]. But in a case of adding a dither signal, equation (13) doesn't match for exact calculation. Fig. 5 shows the photodetector output with dither signal.

Dithering is a widely used method in gyroscopes to overcome the deadzone where it can't detect any rate [12-14]. The square-wave dither signal used in FOG changes the photodetector output power because it generates the additional phase shift in feedback modulation. In order to calculate the error considering dither amplitude $\Delta \phi_{\text {dith }}$, we should apply $\Delta \phi_{m}+\Delta \phi_{\text {dith }}$ to equation (12), instead of $\Delta \phi_{m}$ :

$$
\begin{aligned}
P_{\text {error }} & =P_{2}-P_{1}=P_{0} \sin \left(\Delta \phi_{m} \pm \Delta \phi_{\text {dith }}\right) \sin \delta \\
& \approx \cos \Delta \phi_{\text {dith }} \cdot \delta
\end{aligned}
$$

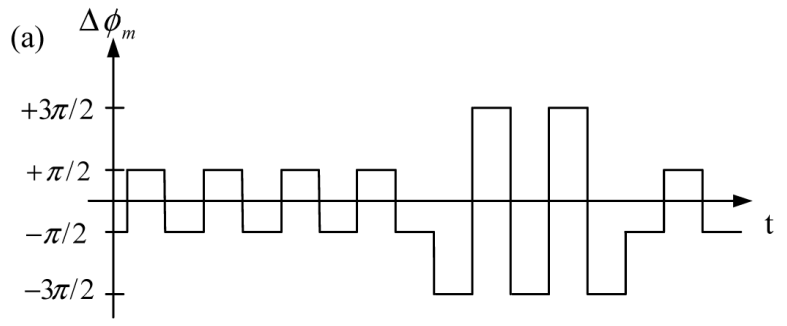

(b)
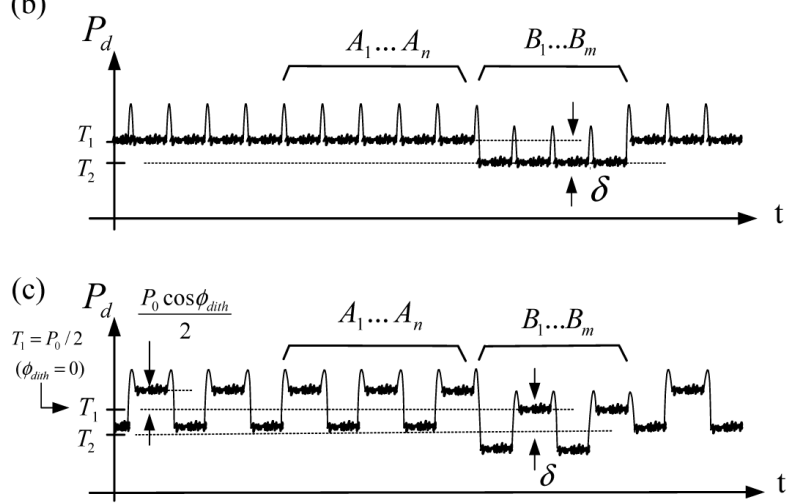

FIG. 5. (a) Phase modulation steps (b) photodetector output without dithering (c) photodetector output with dithering.

This equation has a nonlinear term $\cos \triangle \phi_{\text {dith }}$ that should be cancelled or compensated. The modulation amplitude error without dithering (Fig. 5(b)) can be successfully calculated by equation (13) without additional error. But the modulation amplitude error with dithering can include a residual error induced by dither amplitude, $\Delta \phi_{\text {dith }}$.

The average of photodetector output for even steps $B_{m}$ results in the same as $T_{2}$ because the total average of $\triangle \phi_{\text {dith }}$ is cancelled. But if steps have odd number like $A_{n}$, the additional error occurs. Assume that the number of photodetector output $A_{n}$ is 5 during $\pm \pi / 2$ step as shown in Fig. 5(c). The average is calculated as :

$$
\begin{aligned}
& \text { average }=\frac{1}{5} \sum_{n=1}^{5} A_{n} \\
& =\frac{1}{5}\left\{\left(T_{1}+K\right)+\left(T_{1}-K\right) \ldots\left(T_{1}+K\right)\right\} \\
& =T_{1}+\frac{K}{5}=T_{1}+\frac{P_{0} \cos \Delta \phi_{\text {dith }}}{10} \neq T_{1}
\end{aligned}
$$

where $K$ is $P_{0} \frac{\cos \Delta \phi_{\text {dith }}}{2} ; T_{1}$ is the average of $A_{n} ; T_{2}$ is the average of $B_{m}$

In order to remove the above additional error term $P_{0} \cos \Delta \phi_{\text {dith }} / 10$, let's define $S_{\Delta \phi_{m}}$ as the sum of photodetector outputs and $D_{\Delta \phi_{m}}$ as the difference from the first output to sum of following outputs. By subtracting $D_{\Delta \phi_{m}}$ from $S_{\triangle \phi_{m}}$, we can remove the additional error as below : 


$$
\begin{aligned}
S_{\frac{\pi}{2}} & =\left(T_{1}+K\right)+\left(T_{1}-K\right)+\left(T_{1}+K\right)+\ldots \\
& =n T_{1}+K \\
D_{\frac{\pi}{2}} & =\left(T_{1}+K\right)-\left\{\left(T_{1}-K\right)+\left(T_{1}+K\right)+\ldots\right\} \\
& =\left\{(2-n) T_{1}+K\right\} \\
S_{\frac{\pi}{2}} & -D_{\frac{\pi}{2}}=(2 n-2) T_{1}
\end{aligned}
$$

where $n$ is the odd number ; $\triangle \phi_{m}=\pi / 2$.

The variables $n, S_{\Delta \phi_{m}}$, and $D_{\Delta \phi_{m}}$ are known values which can be extracted from the digital logic. The average value $T_{1}$ is calculated by simply dividing $(2 n-2)$, as shown in Table 1 . The same rule for $\pm 3 \pi / 2$ can be applied.

The modulation amplitude error calculated from Table 1 can be compensated by the signal control loop shown in Fig. 6. The modulation amplitude controlled by PWM generator which transforms the digital value to pulse can adjust the range of $\mathrm{D} / \mathrm{A}$ converter. A control loop model expressed in Laplace transform is shown in Fig. 7.

where $K_{o p}$ is the open loop gain ; $K_{i}$ is the integrator gain for stabilizing the loop ; $K_{a m p}$ is the gain of the D/A converter ; $K_{\text {mod }}$ is the modulation coefficient ; $G(s)$ is the controller for closing the loop; $K_{m}$ is the gain for amplitude compensation.

When the gyroscope operates closed-loop mode in rate, the scale factor is not related to the open loop gain any more if the open loop gain is sufficiently large. The relationship between the control loop transfer function and scale factor can be expressed as :

$$
\text { Scale Factor }(S . F)=\left.\frac{C(s)}{\Omega(s)}\right|_{s=0} \approx \frac{K_{O S F}}{K_{a m p} K_{\bmod }}
$$

The scale factor of the gyroscope depends on three factors, $K_{O S F}, K_{a m p}$, and $K_{\text {mod }}$. Among them, the temperature dependency of $K_{O S F}$ and $K_{a m p}$ can be kept below a few ppm by wavelength stabilization and precision gain reference

TABLE 1 . The average of steps during $\pm \pi / 2$

\begin{tabular}{lcc}
\hline $\begin{array}{c}\text { Type of number in } \\
n \text { steps }\end{array}$ & even & odd \\
\hline Average of steps & $\frac{1}{n} S_{\Delta \phi_{m}}$ & $\frac{1}{(2 n-2)}\left(S_{\Delta \phi_{m}}-D_{\Delta \phi_{m}}\right)$ \\
$n=2,4,6, \cdots$ & $n=3,5,7, \cdots$ \\
\hline$S_{\Delta \phi_{m}}=\sum_{i=1}^{n} \frac{P_{0}}{2}\left\{1+\cos \left(\Delta \phi_{m}+\delta+(-1)^{i} \Delta \phi_{\text {dith }}\right)\right\}$ \\
$D_{\Delta \phi_{m}}=\frac{P_{0}}{2}\left\{1+\cos \left(\Delta \phi_{m}+\delta+(-1)^{1} \Delta \phi_{\text {dith }}\right)\right\}$ \\
$\quad-\sum_{i=2}^{n} \frac{P_{0}}{2}\left\{1+\cos \left(\Delta \phi_{m}+\delta+(-1)^{i} \Delta \phi_{\text {dith }}\right)\right\}$
\end{tabular}

$i$ : phase modulation sequences circuits. So, the temperature dependency of modulation amplitude coefficient $d K_{\text {mod }} / d T$ is dominantly proportional to $d n_{\text {eff }} / d T$ from equation (9), and directly influences the scale factor of the gyroscope.

Assume $G(s)$ has a single-pole integrator. The input-output transfer function for the amplitude control loop is as below :

$$
\frac{C_{m}(s)}{\Delta \phi_{m}(s)}=\frac{0.5 P_{0} K_{\mathrm{mod}} K_{a m p} G(s)}{1+0.5 P_{0} K_{\mathrm{mod}} K_{a m p} G(s)}=K \cdot \frac{1}{\tau s+1}
$$

where $\tau$ is the time constant for the loop ; $K$ is the DC gain.

When the loop is closed, the output $C_{m}(s)$ has a desired value depending on temperature change and the feedback compensation $\alpha$, shown in Fig. 7, is performed as follows :

$$
\begin{array}{r}
\alpha=K_{a m p} C(s) \cdot\left(1+K_{m} C_{m}(s)\right) \\
\text { IV. TEST RESULTS }
\end{array}
$$

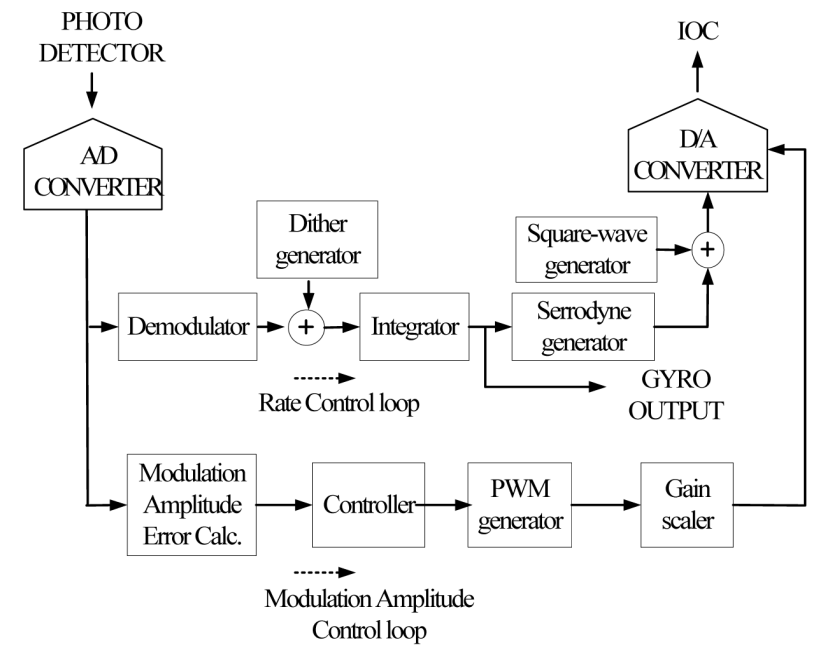

FIG. 6. Signal processing block diagram for phase modulation amplitude control.

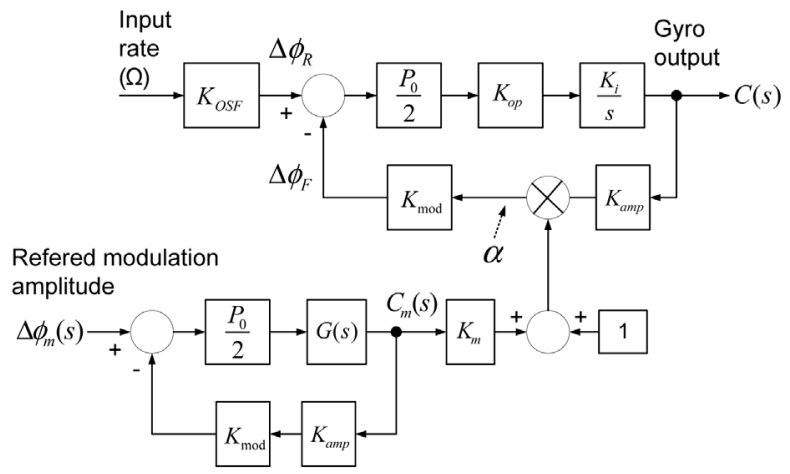

FIG. 7. Modeling of phase modulation amplitude control loop. 
The optical head and signal processing modules to verify the effect of amplitude control were implemented on prototype FOG. The optical head was made with Polarization Maintaining (PM) fiber, $1550 \mathrm{~nm}$ broadband source, IOC, $2 \times 2$ coupler, and photodetector. A $900 \mathrm{~m}$ PM fiber with a low birefringence and a small cladding diameter was chosen for good bias performance, wound in fiber spool with quadrupolar winding for reducing the temperature and vibration errors. A broadband source was made with 980 nm-pump laser diode, WDM, and coupler. This configuration promises good stability in power and wavelength. A push-pull modulator with long electrodes on the IOC was designed for high modulation efficiency. A photodetector with low dark noise and low capacitance was chosen for a low noise, high efficiency amplifier design. Special care should be taken in designing the photoamplifier as a small current below a few nano-ampere(nA) corresponding to small input rate can flow on circuits. The signal processing module includes constant current driving circuits for supplying constant power to the laser diode, digital modulationdemodulation logic, control logics for rate and modulation amplitude control, and DSP unit for compensation and digital communication. The optical head and signal processing module are shown in Fig. 8.

A Figure 9 shows a simulation result which compares a simple averaging method with the suggested method. In an amplitude open-loop mode, the amplitude error was increased up to $0.6 \%$ as dither amplitude was changed from $9 \mathrm{deg} / \mathrm{sec}$ to $15 \mathrm{deg} / \mathrm{sec}$, while the suggested method was unchanged. This error can lead to a bias drift or scale factor error, even though the error goes to zero in an amplitude closed-loop mode.

On the basis of this simulation result, the suggested method was adopted on signal processing module. Experimental results from Fig. 10 to Fig. 13 show the effect of amplitude

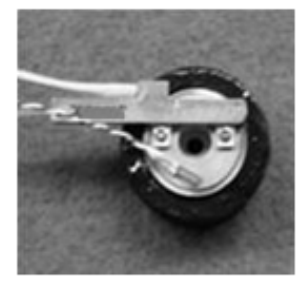

(a) fiber spool

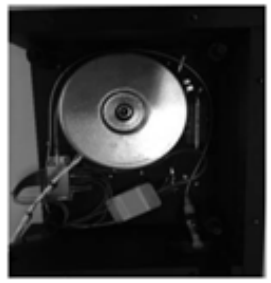

(b) optical head assembly

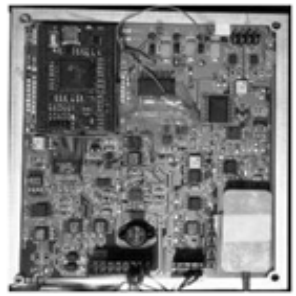

(c) signal processing module

FIG. 8. Optical head and signal processing module for prototype FOG. control.

The error without amplitude control shown in Fig. 10 is approximately 26,000 ppm(peak-to-peak) over full temperature range, which corresponds to $325 \mathrm{ppm} /{ }^{\circ} \mathrm{C}$, a reasonable value for the typical temperature dependency of $r_{e f f}$ as stated earlier in equation (9). Relatively the error with amplitude control is significantly reduced.

Figure 11 shows the relationship between amplitude control and scale factor. The scale factor is not only related to modulation amplitude $K_{\bmod }$ but can be affected by optical scale factor $K_{O S F}$, according to equation (17). The variation of $K_{\text {amp }}$ and $K_{\mathrm{mod}}$ can be compensated by amplitude control loop but the variation of $K_{O S F}$ cannot be controlled. In order to find how much the modulation amplitude error can affect the scale factor, the amplitude control loop was tested without rotation (upper graph) and next, the scale factor was tested in rotation (lower graph). The upper graph shows the residual error when the amplitude is controlled, existing unexpected temperature drift by modulator

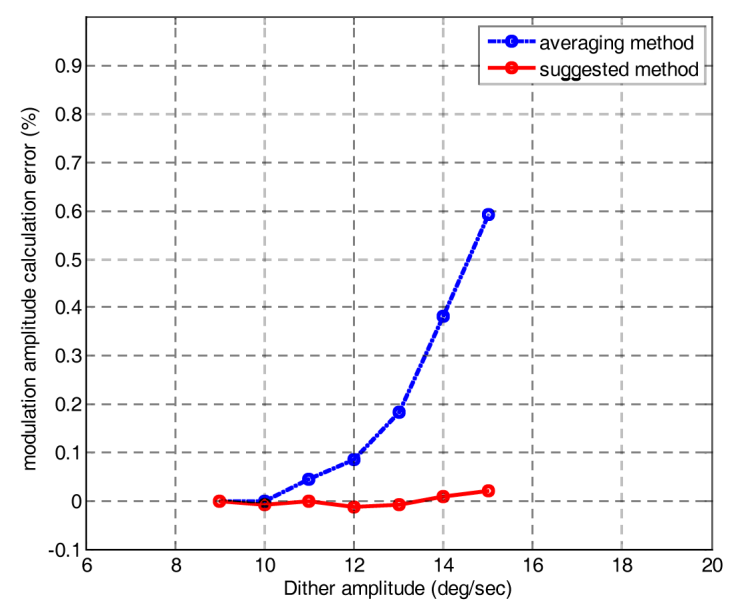

FIG. 9. Modulation amplitude calculation error vs dither amplitude (simulation).

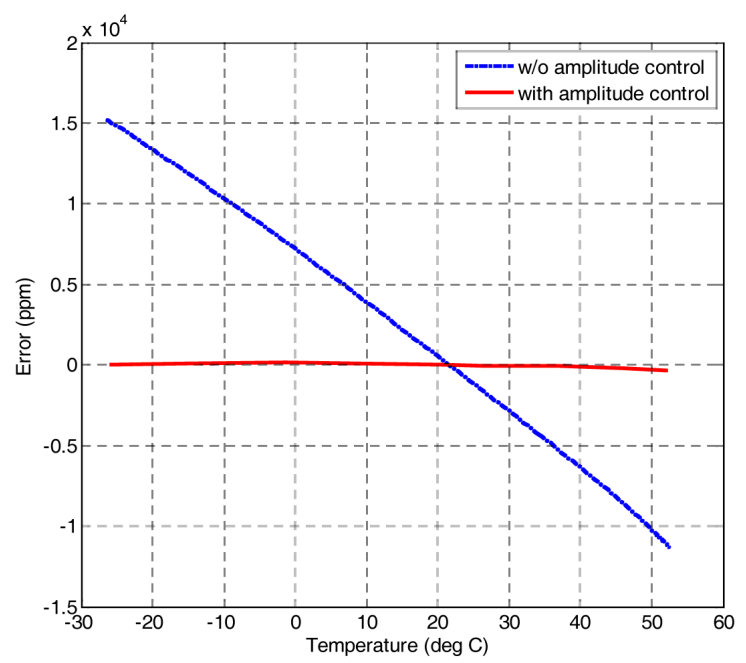

FIG. 10. Modulation amplitude error (measurement). 

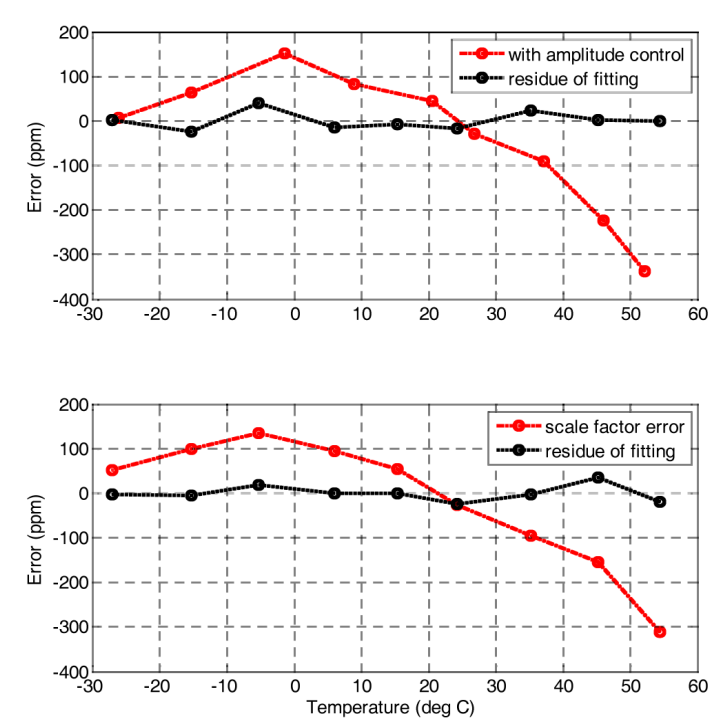

FIG. 11. Scale factor error (measurement).

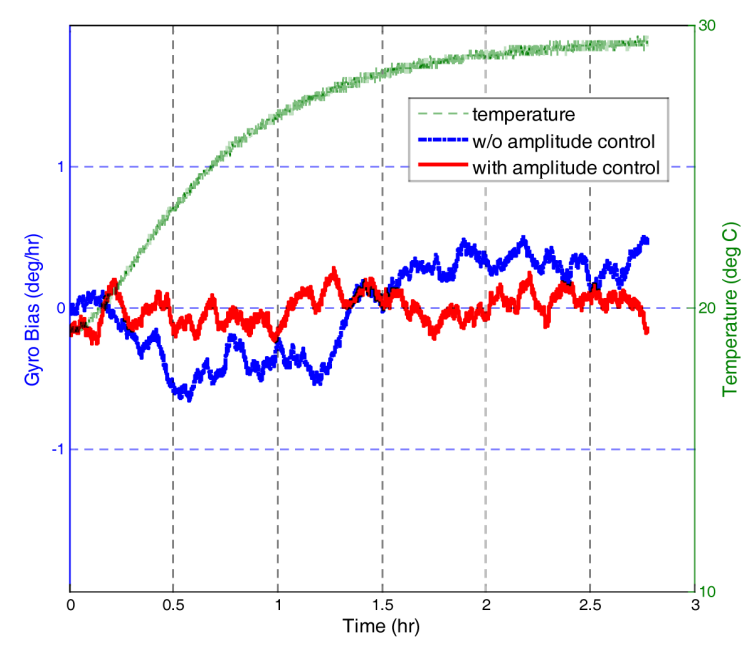

FIG. 12. Bias instability (measurement).

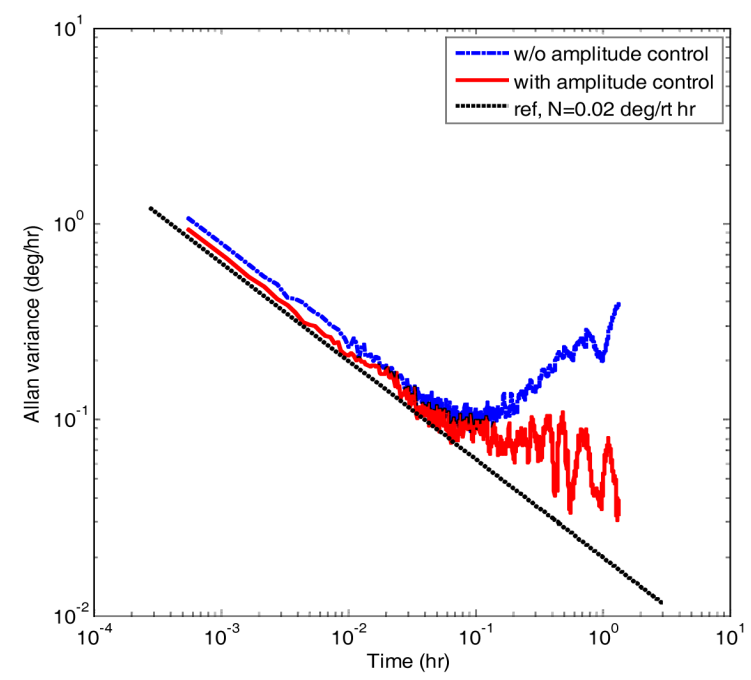

FIG. 13. Allan variance (measurement). or electronics. The lower graph has a similar curve to upper's and the peak-to-peak values of both errors are almost same, $489 \mathrm{ppm}$ and $444 \mathrm{ppm}$, respectively, and likewise, the residual errors by third order fitting are 64 ppm and $60 \mathrm{ppm}$, respectively. This result shows the scale factor is closely related to modulation amplitude. The scale factor residual error also corresponds to a sufficiently low value, acceptable for a navigation system.

The bias stability, one of the important parameters in determining gyroscope performance, was tested at room temperature. Even in room environment, the temperature is varied approximately $10^{\circ} \mathrm{C}$ because the electronics mounted on the inner side of the gyro assembly heats up optical components. In Fig. 12, the bias instability without amplitude control is $0.32 \mathrm{deg} / \mathrm{hr}$ ( 1 sigma), changed along the temperature variation, while it is reduced to $0.1 \mathrm{deg} / \mathrm{hr}$ in an amplitude control. The Allan variance in Fig. 13 shows the difference of long-term bias instability more clearly. The Allan variance in an amplitude control is about 0.03 $\mathrm{deg} / \mathrm{hr}$, improved three times than without control (The bottom line of datum indicates the Allan variance value)

\section{CONCLUSION}

In this paper, we have discussed the control method of phase modulation amplitude in dithered closed-loop FOG. The origins induced by the modulation amplitude error have been investigated in terms of the effective electro-optic coefficient, pointing out the predominant factor of the scale factor error in gyroscope. The solution to suppressing the modulation amplitude error was proposed, enabling to the exact calculation in a square-wave dithering. We have shown that the control loop effectively eliminated the modulation amplitude error and was successfully implemented on prototype FOG, resulting in improved performances.

\section{ACKNOWLEDGMENT}

This study was partially supported by the National Research Foundation of Korea(NRF) grant funded by the Korea government(MEST)(No. 2011-0027689) and a grant from the Kunsan National University's Long-term Overseas Research Program in the year 2011.

\section{REFERENCES}

1. H. C. Lefevre, "Fundamentals of the interferometric fiber-optic gyroscope," Proc. SPIE 2837, 2-17 (1996).

2. I. R. Edu, R. Obreja, and T. L. Grigorie, "Current technologies and trends in the development of gyros used in navigation applications - a review," in Proc. The $5^{\text {th }}$ WSEAS International Conference on Communications and Information Technology (Stevens Point, Wisconsin, USA, 
2011), pp. 63-68.

3. V. C. Lefevre and W. R. Lindberg, "Fiber optic gyroscopes and the accomplishments of the DARPA-sponsored GPS guidance package program,” Proc. SPIE 6314, 63140B1-9 (2006).

4. T. Buret, D. Ramecourt, and F. Napolitano, "From space qualified fiber optic gyroscope to generic fiber optic solutions available for space application," in Proc. The $7^{\text {th }}$ International Conference on Space Optics (Toulouse, France, Oct. 2008), pp. 14-17.

5. G. A. Sanders, B. Szafraniec, R. Y. Liu, C. Laskoskie, L. Standjord, and G. Weed, "Fiber optic gyros for space, marine and aviation applications," Proc. SPIE 2837, 61-71 (1996).

6. B. Moslehi, R. Yahalom, L. Oblea, F. Faridian, R. Black, J. Ha, and M. Berarducci, "Low-cost and compact fiber-optic gyroscope with long-term stability," in Proc. IEEE Aerospace Conference (Big Sky, Montana, USA, 2011), pp. 1-9.

7. J. P. Salvestrini, L. Guilbert, M. Fontana, M. Abarkan, and S. Gille, "Analysis and control of the DC drift in $\mathrm{LiNbO}_{3}-$ based Mach-Zehnder modulators," J. Lightwave Technol. 2, 1522-1534 (2011).

8. J. D. Zook, D. Chen, and G. N. Otto, "Temperature dependence and model of the electro-optic effect in $\mathrm{LiNbO}_{3}$," Appl. Phys. Lett. 11, 159-161 (1967).

9. L. M. Johnson, F. J. Leonberger, and G. W. Pratt, "Integrated optical temperature sensor," Appl. Phys. Lett. 41, 134-136 (1982).

10. H. Y. Shen, H. Xu, Z. D. Zeng, W. X. Lin, R. F. Wu, and G. F. Xu, "Measurement of refractive indices and thermal refractive-index coefficients of $\mathrm{LiNbO}_{3}$ crystal doped with 5 mol. \% MgO," Appl. Opt. 31, 6695-6697 (1992).

11. F. Sun, L. Wang, and G. Wang, "Study on the drift of modulated phase in interference fiber optic gyroscope," Journal of Computers 5, 394-400 (2010).

12. G. A. Pavlath, "Closed-loop fiber optic gyros," Proc. SPIE 2837, 46-60 (1996).

13. G. Spahlinger, M. Kemmler, M. Ruf, M. Ribes, and S. Zimmermann, "Error compensation via signal correlation in high precision closed-loop fiber optic gyros," Proc. SPIE 2837, 218-227 (1996).

14. R. A. Kovacs, "Fiber optic gyroscope with reduced nonlinearity at low angular rates," in Proc. $21^{\text {st }}$ Annual AAS Guidance and Control Conference (Boston, MA, USA, 1998), AAS 98-043. 